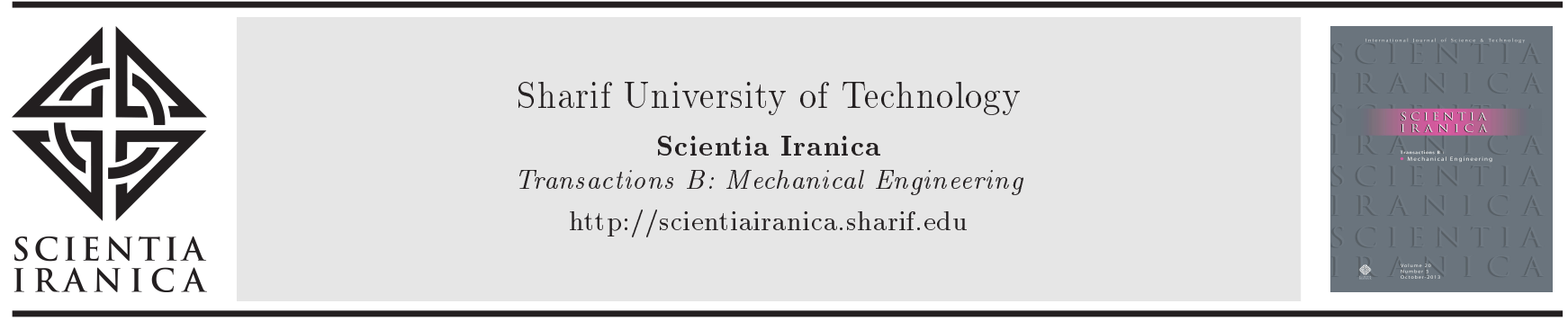

\title{
Chaos and bifurcation in nonlinear in-extensional rotating shafts
}

\author{
S.A.A. Hosseini* \\ Department of Mechanical Engineering, Faculty of Engineering, Kharazmi University, Mofatteh Avenue, Tehran, P.O. Box \\ 15719-14911, Iran.
}

Received 19 September 2017; received in revised form 7 December 2017; accepted 8 January 2018

\section{KEYWORDS \\ Bifurcation; \\ Chaos; \\ Rotor; \\ In-extensional \\ rotating shaft.}

\begin{abstract}
In this paper, bifurcation and chaotic behavior of in-extensional rotating shafts are investigated. The shaft is modeled as a Rayleigh simply supported beam, spinning with constant rotational speed. Using two-mode Galerkin truncation, the partial differential equations of motion are discretized and, then, with the aid of numerical simulations, the dynamical behavior of the rotating shaft is studied. Using tools from nonlinear dynamics, such as time history, bifurcation diagram, Poincaré map, Lyapunov exponents, and amplitude spectrum, a comprehensive analysis is made to characterize the complex behavior of the rotating shaft. Periodic (synchronous), quasi-periodic, chaotic, and transient chaotic responses are observed in the neighborhood of the second critical speed. The effect of rotary inertia and damping on the dynamics of the rotating shaft is considered. It is shown that the chaotic response is possible for a shaft with weak nonlinearity without the existence of any internal resonance.
\end{abstract}

(C) 2019 Sharif University of Technology. All rights reserved.

\section{Introduction}

Due to the high-speed performance of newly developed rotating machinery, the dynamical prediction and analysis of these equipment are necessary. To simplify the analysis, researchers often try to use the linear analysis. But, application of nonlinear analysis is sometimes inevitable. Many phenomena should be described with nonlinear equations which are not explainable with linear analysis. Analysis of chaotic dynamics of rotating machines has been an important topic in rotor-dynamics area in recent years. Ishida [1] reviewed the research on the nonlinear vibration and chaos in rotordynamics field. That paper covered the nonlinearity in restoring and damping forces such as clearances in bearings, squeeze film dampers, oil films

*. Tel.: +982634569555

E-mail address: ali.hosseini@khu.ac.ir in journal bearings, magnetic forces, seals, frictions, and stiffening effect in rotating shaft systems. In one of the early experiments in chaotic motion of rotor systems, Ehrich [2] observed period doubling routes to chaos in a rotor with dead-band nonlinearity. Up to now, many studies have been carried out in study of chaotic vibration in rotor-dynamics; for example, chaotic responses due to rub-impacts in rotors have been studied in references [3-17] and the chaotic dynamics of rotors due to effects of bearing has been investigated in papers [18-25]. Most of these papers treated the rotor with strong nonlinearity. However, in practical rotor-dynamics, these types of nonlinearity occur in rare cases.

Yamamoto and Ishida [26] pointed out that the chaotic vibration in a rotor with weak nonlinearity was possible when the effect of gyroscopic moment was small. Indeed, when the shaft is slender and the gyroscopic effect is small, internal resonances of type 1: (-1) may occur due to nonlinear asymmetry, and this may in turn lead to a chaotic response. Inoue and Ishida [27] 
investigated chaotic vibration and internal resonance phenomena in rotor systems with weak nonlinearity by using theoretical and experimental methods. They found a period-doubling route to chaos. Nagasaka et al. [28] used a continuous rotor with geometrical weak nonlinearity to study the chaotic behavior of a rotating shaft. The nonlinearity was due to the elongation of the shaft, which was induced from restraining the shaft supports in axial direction. Since the shaft was horizontal, the asymmetrical nonlinearity appeared as a result of shifting of equilibrium position. The shaft was very slender, and so the gyroscopic moment was very small.

Using a nonlinear in-extensional shaft model, it is shown that the chaotic response can occur in a continuous rotating shaft with weak symmetric geometrical nonlinearity, even for a fairly large amount of gyroscopic moments. This is in contrast to the assertion of $[26,27]$. In these references, the authors stated that the chaos occurred in rotors with strong nonlinearity or in rotors with weak nonlinearity, but in the presence of internal resonance. Internal resonances occur when the gyroscopic moment is small. In the present paper, the occurrence of chaos in an in-extensional rotor is shown without the existence of the above factors. In other words, it is shown that the chaotic response is possible for a shaft with weak nonlinearity without the existence of any internal resonance. It is observed that the chaotic motion occurs in the rotor for any value of rotary inertia and it is not necessary that the rotor should have a small gyroscopic moment with asymmetric nonlinearity $[26,27]$.

In previous articles [29-32], dynamical behavior of nonlinear in-extensional rotating shafts was studied by the author. In those papers, the periodic response of the shaft was considered and other types of shaft motion, like quasi-periodic and chaotic responses, were not studied. Therefore, some questions remained: Does an in-extensional rotating shaft have a chaotic behavior? In what rotational speed? What is the characteristic of the response? etc. The present paper tries to answer these questions. This article is a continuation of the author's previous papers [29$32]$, in which bifurcation and chaotic behavior of the rotor system were investigated. The shaft is modeled as a Rayleigh simply supported beam, spinning with constant rotational speed. Since the dynamics of the rotating shaft is studied in the neighborhood of the second critical speed, two-mode Galerkin truncation is applied to discretize the partial differential equations of motion into eight first-order ordinary differential equations in two transverse planes. Using fourthorder Runge-Kutta method (please see the Appendix for the flowchart of the method), with the aid of time history, bifurcation diagram, Poincaré map, Lyapunov exponents, and amplitude spectrum, a comprehensive analysis is made to investigate the dynamical behavior of the rotating shaft. When the rotational speed is near the second whirling mode, periodic, quasi-periodic, and chaotic motions as well as transient chaos are observed. The chaotic motion occurs in a vast range of parameters. The effect of rotary inertia (gyroscopic moment) and damping on the dynamics of the rotating shaft is considered.

\section{Equations of motion}

Figure 1 shows a schematic of a rotating shaft before deformation. The frame $X-Y-Z$ is an inertial coordinate, in which axis $X$ is along the centerline of the undeformed shaft. The length of the undeformed shaft is $l$. The $x-y-z$ axes constitute a local coordinate. The axes are attached to the centerline of the deformed shaft at position $x$. Displacements of a particle in arbitrary location $x$ along $X, Y$, and $Z$ axes are $u(x, t), \nu(x, t)$, and $w(x, t)$, respectively. The following assumptions are employed:

1. The shaft has uniform circular cross section and is isotropic;

2. Shaft spins around longitudinal axis $X$ with constant speed $\Omega$;

3. The effect of gravity is neglected;

4. The warping effects are neglected;

5. The rotary inertia and gyroscopic effects are considered;

6. The rotating shaft is simply supported;

7. Support $\mathbf{O}$ of the shaft is fixed, but support $\mathbf{O}^{\prime}$ is movable along the $X$ axis (Figure 1). This assumption implies that the stretching effect is negligible. This situation is more realistic than that in some previous works in which nonlinearity was due to the stretching of the shaft centerline, e.g., [28];

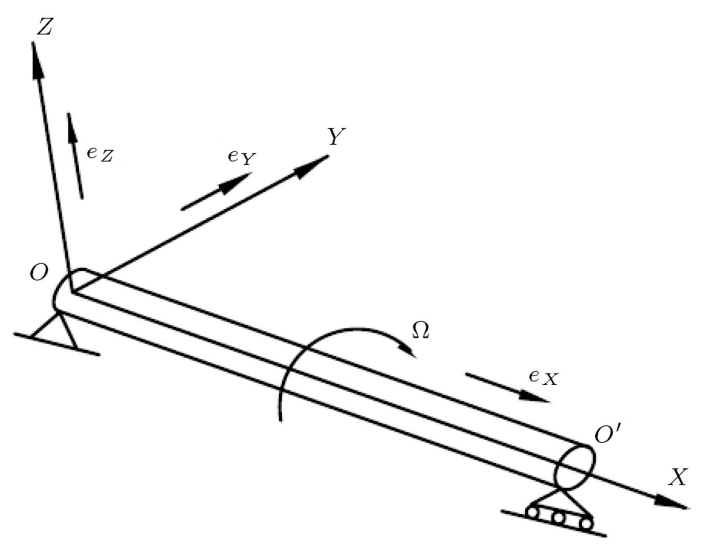

Figure 1. Schematic of a rotating shaft and inertial coordinate $X-Y-Z$. 
8. The only dissipating mechanism in the system is external viscose damping;

9. The amplitude of vibration is large and shortening effect due to in-extensibility assumption is considered [33];

10. The excitation is due to eccentricity in the shaft.

First, the following dimensionless quantities are defined:

$$
\begin{aligned}
& x^{*}=x / l, \quad \nu^{*}=\nu / l, \quad w^{*}=w / l, \\
& t^{*}=\sqrt{\frac{D_{22}}{m l^{4}}} t, \quad c^{*}=c l^{2} / \sqrt{m D_{22}}, \\
& I_{2}^{*}=I_{2} /\left(m l^{2}\right), \quad \Omega^{*}=\sqrt{\frac{m l^{4}}{D_{22}}} \Omega, \\
& e_{\varsigma}^{*}\left(x^{*}\right)=e_{\varsigma}(x) / l, \quad e_{\xi}^{*}\left(x^{*}\right)=e_{\xi}(x) / l,
\end{aligned}
$$

where $t$ is time, $D_{22}$ is transverse rigidity, $m$ is mass per unit length of the shaft, $c$ is external damping coefficient, $I_{2}$ is diametrical mass moment of inertia, and $e_{\varsigma}(x)$ and $e_{\xi}(x)$ are eccentricity distributions measured with respect to axes $y$ and $z$, respectively.

Applying the above assumption and using dimensionless quantities (1), after dropping the asterisks, the equations of motion governing the vibration of an inextensional rotating shaft can be written as [29]:

$$
\begin{aligned}
& \ddot{\nu}+\nu^{\prime} \int_{0}^{x}\left(\dot{\nu}^{\prime 2}+\ddot{\nu}^{\prime} \nu^{\prime}+\dot{w}^{\prime 2}+\ddot{w}^{\prime 2} w^{\prime}\right) d x \\
& +c \dot{\nu}-I_{2}\left(2 \Omega \dot{w}^{\prime \prime}+\ddot{\nu}^{\prime \prime}\right)+\nu^{\prime 2} \nu^{(I V)}+\nu^{\prime} w^{(I V)} w^{\prime} \\
& +3 w^{\prime \prime \prime} w^{\prime \prime} \nu^{\prime}+\nu^{\prime \prime} w^{\prime \prime 2}+\nu^{\prime \prime 3}+\nu^{(I V)}+w^{\prime \prime \prime} \nu^{\prime \prime} w^{\prime} \\
& +4 \nu^{\prime} \nu^{\prime \prime} \nu^{\prime \prime \prime}+\nu^{\prime \prime} \int_{l}^{x} \int_{0}^{x}\left(\dot{\nu}^{\prime 2}+\ddot{\nu}^{\prime} \nu^{\prime}+\dot{w}^{\prime 2}+\ddot{w}^{\prime} w^{\prime}\right) d x d x \\
& =\Omega^{2}\left[e_{\xi}(x) \cos \Omega t-e_{\varsigma}(x) \sin \Omega t\right], \\
& \ddot{w}+w^{\prime} \int_{0}^{x}\left(\dot{\nu}^{\prime 2}+\ddot{\nu}^{\prime} \nu^{\prime}+\dot{w}^{\prime 2}+\ddot{w}^{\prime} w^{\prime}\right) d x+c \dot{w} \\
& +I_{2}\left(2 \Omega \dot{\nu}^{\prime \prime}-\ddot{w}^{\prime \prime}\right)+w^{\prime \prime} \nu^{\prime \prime 2}+w^{(I V)}+\nu^{\prime} \nu^{\prime \prime \prime} w^{\prime \prime} \\
& +3 w^{\prime} \nu^{\prime \prime} \nu^{\prime \prime \prime}+w^{\prime} \nu^{\prime} \nu^{(I V)}+w^{\prime 2} w^{(I V)}+w^{\prime \prime 3} \\
& +4 w^{\prime} w^{\prime \prime} w^{\prime \prime \prime}+w^{\prime \prime} \int_{l}^{x} \int_{0}^{x}\left(\dot{\nu}^{\prime 2}+\ddot{\nu}^{\prime} \nu^{\prime}+\dot{w}^{\prime 2}\right. \\
& \left.+\ddot{w}^{\prime} w^{\prime}\right) d x d x=\Omega^{2}\left[e_{\xi}(x) \sin \Omega t+e_{\varsigma}(x) \cos \Omega t\right] .(2)
\end{aligned}
$$

The boundary conditions are:

$$
\nu=0, \quad \nu^{\prime \prime}=0, \quad w=0, \quad w^{\prime \prime}=0
$$

$$
\text { at } x=0 \text { and } x=l \text {. }
$$

Derivation of the above equations has been explained thoroughly in [29], and it is not repeat here. Eq. (2) includes effect of nonlinear curvature as well as nonlinear inertia.

The partial differential equation of motion is discretized using two-mode Galerkin truncation as:

$$
\begin{aligned}
& \nu(x, t)=\phi_{1}(x) \nu_{1}(t)+\phi_{2}(x) \nu_{2}(t), \\
& w(x, t)=\phi_{1}(x) w_{1}(t)+\phi_{2}(x) w_{2}(t),
\end{aligned}
$$

where $\phi_{j}(x)=\sqrt{2} \sin j \pi x(j=1,2)$ is the linear mode shape of the shaft [29]. Since the dynamics of the rotating shaft is studied in the neighborhood of the second critical speed, two-mode Galerkin truncation is applied.

Substituting Eq. (4) into Eq. (2), taking the inner product of each equation with its corresponding mode shape, and using the orthogonality properties of the mode shapes, one can obtain the following discretized equations of motion:

$$
\begin{aligned}
& \pi^{2}\left(\frac{20}{9} \nu_{1}^{2}+\frac{4}{3} \pi^{2} \nu_{1} \nu_{2}+\frac{35}{36} \nu_{2} \nu_{1}+\frac{80}{9} \nu_{2}^{2}\right) \ddot{\nu}_{2} \\
& +\left(1+\frac{40}{9} \pi^{2} \nu_{2} \nu_{1}-\frac{3}{8} \nu_{1}{ }^{2} \pi^{2}+\frac{20}{9} \pi^{2} \nu_{2}{ }^{2}\right. \\
& \left.+I_{2} \pi^{2}+\frac{1}{3} \nu_{1}^{2} \pi^{4}\right) \ddot{\nu}_{1}+\pi^{2}\left(\frac{20}{9} \nu_{2} w_{1}\right. \\
& \left.+\frac{20}{9} \nu_{1} w_{2}+\frac{1}{3} \pi^{2} \nu_{1} w_{1}+\frac{20}{9} \nu_{2} w_{2}-\frac{3}{8} \nu_{1} w_{1}\right) \ddot{w}_{1} \\
& +\pi^{2}\left(\frac{20}{9} \nu_{2} w_{1}+\frac{4}{3} \pi^{2} \nu_{1} w_{2}-\frac{5}{4} \nu_{1} w_{2}+\frac{80}{9} \nu_{2} w_{2}\right. \\
& \left.+\frac{20}{9} \nu_{1} w_{1}\right) \ddot{w}_{2}+\pi^{2}\left(\frac{80}{9} \nu_{2}+\frac{4}{3} \nu_{1} \pi^{2}-\frac{5}{4} \nu_{1}\right) \\
& \left(\dot{w}_{2}^{2}+\dot{\nu}_{2}^{2}\right)+\frac{40}{9} \pi^{2}\left(\nu_{1}+\nu_{2}\right)\left(\dot{w}_{1} \dot{w}_{2}+\dot{\nu}_{1} \dot{\nu}_{2}\right) \\
& +\pi^{2}\left(-\frac{3}{8} \nu_{1}+\frac{20}{9} \nu_{2}+\frac{1}{3} \nu_{1} \pi^{2}\right)\left(\dot{\nu}_{1}^{2}+\dot{w}_{1}^{2}\right) \\
& +2 I_{2} \Omega \dot{w}_{1} \pi^{2}+\nu_{1} \pi^{4}+c \dot{\nu}_{1}+\pi^{6}\left(w_{1}^{2} \nu_{1}+\nu_{1}^{3}\right) \\
& +20 \pi^{6}\left(\nu_{1} \nu_{2}^{2}+\nu_{2} w_{2} w_{1}\right) \\
& =\Omega^{2}\left[e_{21} \cos \Omega t-e_{11} \sin \Omega t\right],
\end{aligned}
$$$$
\pi^{2}\left(\frac{20}{9} \nu_{1}^{2}+\frac{4}{3} \pi^{2} \nu_{1} \nu_{2}+\frac{35}{36} \nu_{2} \nu_{1}+\frac{80}{9} \nu_{2}^{2}\right) \ddot{\nu}_{1}
$$ 


$$
\begin{aligned}
& +\pi^{2}\left(-\frac{3}{2} \nu_{2}+\frac{80}{9} \nu_{1}+\frac{16}{3} \nu_{2} \pi^{2}\right)\left(\dot{w}_{2}^{2}+\dot{\nu}_{2}^{2}\right) \\
& +\pi^{2}\left(\frac{160}{9} \nu_{2}+\frac{40}{9} \nu_{1}\right)\left(\dot{w}_{1} \dot{w}_{2}+\dot{\nu}_{1} \dot{\nu}_{2}\right) \\
& +8 I_{2} \Omega \pi^{2} \dot{w}_{2}+c \dot{\nu}_{2} \pi^{2}\left(\frac{4}{3} \nu_{2} \pi^{2}-\frac{5}{4} \nu_{2}+\frac{20}{9} \nu_{1}\right) \\
& \left(\dot{\nu}_{1}^{2}+\dot{w}_{1}^{2}\right)+\left(1+\frac{160}{9} \pi^{2} \nu_{2} \nu_{1}-\frac{3}{2} \pi^{2} \nu_{2}^{2}+\frac{16}{3} \pi^{4} \nu_{2}^{2}\right. \\
& \left.+\frac{20}{9} \nu_{1}^{2} \pi^{2}+4 I_{2} \pi^{2}\right) \ddot{\nu}_{2}+\pi^{2}\left(\frac{80}{9} \nu_{2} w_{2}+\frac{20}{9} \nu_{1} w_{1}\right. \\
& \left.-\frac{5}{4} \nu_{2} w_{1}+\frac{4}{3} \pi^{2} \nu_{2} w_{1}+\frac{20}{9} \nu_{1} w_{2}\right) \ddot{w}_{1}+16 \nu_{2} \pi^{4} \\
& +20 \pi^{6}\left(w_{1} w_{2} \nu_{1}+\nu_{1}{ }^{2} \nu_{2}\right)+64 \pi^{6}\left(\nu_{2}^{3}+\nu_{2} w_{2}^{2}\right) \\
& +\left(-\frac{3}{2} \pi^{2} \nu_{2} w_{2}+\frac{80}{9} \pi^{2} \nu_{2} w_{1}+\frac{20}{9} \pi^{2} \nu_{1} w_{1}\right. \\
& \left.+\frac{80}{9} \nu_{1} \pi^{2} w_{2}+\frac{16}{3} \pi^{4} \nu_{2} w_{2}\right) \ddot{w}_{2} \\
& =\Omega^{2}\left[e_{22} \cos \Omega t-e_{12} \sin \Omega t\right] \\
& \pi^{2}\left(\frac{80}{9} w_{2}^{2}+\frac{4}{3} w_{1} \pi^{2} w_{2}+\frac{20}{9} w_{1}^{2}+\frac{35}{36} w_{2} w_{1}\right) \ddot{w}_{2} \\
& +\left(1-\frac{3}{8} \pi^{2} w_{1}^{2}+\frac{1}{3} \pi^{4} w_{1}^{2}+\frac{20}{9} w_{2}^{2} \pi^{2}+I 2 \pi^{2}\right. \\
& \left.+\frac{40}{9} \pi^{2} w_{2} w_{1}\right) \ddot{w}_{1}+\pi^{2}\left(\frac{20}{9} \nu_{2} w_{1}+\frac{20}{9} \nu_{1} w_{2}\right. \\
& \left.+\frac{1}{3} \pi^{2} \nu_{1} w_{1}+\frac{20}{9} \nu_{2} w_{2}-\frac{3}{8} \nu_{1} w_{1}\right) \ddot{\nu}_{1} \\
& +\pi^{2}\left(\frac{80}{9} \nu_{2} w_{2}+\frac{20}{9} \nu_{1} w_{1}-\frac{5}{4} \nu_{2} w_{1}\right. \\
& \left.+\frac{4}{3} \pi^{2} \nu_{2} w_{1}+\frac{20}{9} \nu_{1} w_{2}\right) \ddot{\nu}_{2}+\pi^{2}\left(-\frac{5}{4} w_{1}+\frac{80}{9} w_{2}\right. \\
& \left.+\frac{4}{3} w_{1} \pi^{2}\right)\left(\dot{w}_{2}^{2}+\dot{\nu}_{2}^{2}\right)+\pi^{2}\left(\frac{40}{9} w_{2}+\frac{40}{9} w_{1}\right) \\
& \left(\dot{\nu}_{1} \dot{\nu}_{2}+\dot{w}_{1} \dot{w}_{2}\right)+\pi^{2}\left(\frac{20}{9} w_{2}-\frac{3}{8} w_{1}+\frac{1}{3} w_{1} \pi^{2}\right) \\
& \left(\dot{\nu}_{1}^{2}+\dot{w}_{1}^{2}\right)+c \dot{w}_{1}+\pi^{6}\left(w_{1} \nu_{1}^{2}+w_{1}^{3}\right) \\
& +w_{1} \pi^{4}-2 I_{2} \pi^{2} \Omega \dot{\nu}_{1}+20 \pi^{6}\left(w_{1} w_{2}^{2}+w_{2} \nu_{2} \nu_{1}\right)
\end{aligned}
$$

$$
\begin{aligned}
& =\Omega^{2}\left[e_{21} \sin \Omega t+e_{11} \cos \Omega t\right] \\
& \pi^{2}\left(\frac{80}{9} w_{2}^{2}+\frac{4}{3} w_{1} \pi^{2} w_{2}+\frac{20}{9} w_{1}^{2}+\frac{35}{36} w_{2} w_{1}\right) \ddot{w}_{1} \\
& +\pi^{2}\left(\frac{16}{3} w_{2} \pi^{2}+\frac{80}{9} w_{1}-\frac{3}{2} w_{2}\right)\left(\dot{\nu}_{2}^{2}+\dot{w}_{2}^{2}\right)+c \dot{w}_{2} \\
& +\pi^{2}\left(\frac{160}{9} w_{2}+\frac{40}{9} w_{1}\right)\left(\dot{\nu}_{1} \dot{\nu}_{2}+\dot{w}_{1} \dot{w}_{2}\right)+16 w_{2} \pi^{4} \\
& -8 I_{2} \Omega \pi^{2} \dot{\nu}_{2}+20 \pi^{6}\left(w_{1} \nu_{1} \nu_{2}+w_{1}^{2} w_{2}\right) \pi^{2}\left(-\frac{5}{4} w_{2}\right. \\
& \left.+\frac{20}{9} w_{1}+\frac{4}{3} w_{2} \pi^{2}\right)\left(\dot{\nu}_{1}^{2}+\dot{w}_{1}^{2}\right)+64 \pi^{6}\left(w_{2} \nu_{2}^{2}+w_{2}^{3}\right) \\
& +\left(1+4 I_{2} \pi^{2}-\frac{3}{2} w_{2}^{2} \pi^{2}+\frac{160}{9} \pi^{2} w_{2} w_{1}\right. \\
& \left.+\frac{16}{3} \pi^{4} w_{2}^{2}+\frac{20}{9} \pi^{2} w_{1}^{2}\right) \ddot{w}_{2}+\pi^{2}\left(-\frac{3}{2} \nu_{2} w_{2}\right. \\
& \left.+\frac{80}{9} \nu_{2} w_{1}+\frac{20}{9} \nu_{1} w_{1}+\frac{80}{9} \nu_{1} w_{2}+\frac{16}{3} \pi^{2} \nu_{2} w_{2}\right) \ddot{\nu}_{2} \\
& +\pi^{2}\left(\frac{20}{9} \nu_{2} w_{1}+\frac{4}{3} \pi^{2} \nu_{1} w_{2}-\frac{5}{4} \nu_{1} w_{2}+\frac{80}{9} \nu_{2} w_{2}\right. \\
& \left.+\frac{20}{9} \nu_{1} w_{1}\right) \ddot{\nu}_{1}=\Omega^{2}\left[e_{22} \sin \Omega t+e_{12} \cos \Omega t\right],
\end{aligned}
$$

where:

$$
\begin{aligned}
& e_{1 j}=\int_{0}^{1}\left[\sqrt{2} e_{\varsigma}(x) \sin j \pi x\right] d x, \\
& e_{2 j}=\int_{0}^{1}\left[\sqrt{2} e_{\xi}(x) \sin j \pi x\right] d x, \quad j=1,2 .
\end{aligned}
$$

In the next section, Eq. (5) is used to investigate the dynamics of the shaft in different rotational speeds, especially near the second whirling mode.

\section{Dynamical analysis of the rotating shaft}

In this section, with the aid of numerical simulation, the dynamical behavior of the rotating shaft is considered. Fourth-order one-step Runge-Kutta method is applied to numerically integrate equation (5). In statespace form, Eq. (5) can be written as:

$$
\dot{X}=F(X, \Omega t),
$$

where $X=\left[\begin{array}{llllllll}\nu_{1} & \dot{\nu}_{1} & \nu_{2} & \dot{\nu}_{2} & w_{1} & \dot{w}_{1} & w_{2} & \dot{w}_{2}\end{array}\right]$.

The characteristics of the shaft response are studied by use of different tools from nonlinear dynamics 
such as time-history, Poincaré map, bifurcation diagram, Lyapunov exponent, and amplitude spectrum.

Since Eq. (7) is non-autonomous, construction of the Poincaré map is easy [34]. To create a Poincaré map, the response is sampled with frequency $\Omega / 2 \pi$, and then the result is projected onto a suitable plane. For example, to construct a Poincaré map for the firstmode response in $\nu$-plane, Eq. (7) is integrated and then, it is sampled with frequency $\Omega / 2 \pi$; finally, the values of $\left[\nu_{1} \dot{\nu}_{1}\right]$ are plotted in a diagram. Only steady state response is of interest; therefore, the transient part of solution is discarded and Poincaré map is constructed using steady state solution. To plot the bifurcation diagram, a similar procedure is followed, with one exception that intended displacement (or velocity) is plotted against the control parameter (here, rotational speed).

Lyapunov exponent can be used to detect a chaotic motion. The largest positive Lyapunov exponent is a sign of chaos (sensitivity to initial conditions) and a non-positive one shows regular (nonchaotic) motion [34]. To compute the largest Lyapunov exponent, the procedure explained in [34, page 183] is followed, namely, Eq. (7) and corresponding variational equations are integrated simultaneously, and then the divergence of a trajectory in the neighborhood of reference points is locally computed. Finally, the effects of all local divergences are super-imposed to compute the largest Lyapunov exponent. Again, these computations are applied to the steady state part of the solution.

Numerical simulation to Eq. (5) shows that near the first whirling mode, the shaft response is synchronous, i.e., the shaft whirls with frequency equal to the rotational speed. In contrast, near the second whirling mode, the rotating shaft shows complex dynamical behavior. Thus, the dynamics of the rotating shaft is investigated near the second whirling mode. Specifically, the rotational speed $\Omega$ is assumed to be in the interval (34-42). Eq. (5) is simulated and the equations are in dimensionless form, so all parameters and variables used as well as results, which are presented in this section, are in dimensionless form. In addition, all initial conditions are set to zero in all simulations.

\subsection{A slender rotating shaft with light damping}

Two important parameters in dynamical behavior of the shaft are eccentricity $e_{i j} i, j=1,2$, and rotational speed $\Omega$. Instead of considering the effects of theses parameters separately, they are present in a diagram and the shaft response is characterized using tools from nonlinear dynamics (e.g., Lyapunov exponent). Figure 2 shows such a diagram, in which $c=0.03$ and $I_{2}=0.000625$. Numerical simulation shows that with the above data, the response of the shaft near the first

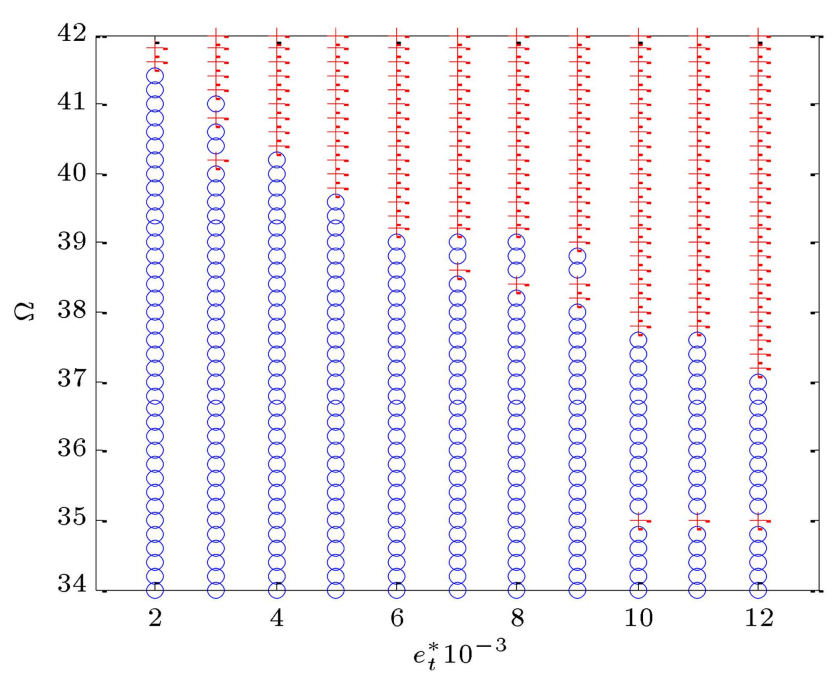

Figure 2. Different types of responses; $I_{2}=0.000625$, $c=0.03$. O: periodic, + : chaotic.

whirling mode is periodic (this result is not presented here). The complex behavior of the shaft appears near the second whirling mode, and so Figure 2 is plotted in this range. Rotational speed increases from $\Omega=34$ with step 0.2 up to $\Omega=42$. Eccentricity increases from $e_{t}=e_{11}=e_{12}=0.002$ with step 0.001 up to $e_{t}=e_{11}=e_{12}=0.012$. In all cases, it is assumed that the eccentricity is in one plane, so $e_{21}=e_{22}=0$. Figure 2 gives a complete pattern of different types of motion in different values of eccentricity and rotational speed. Periodic and chaotic motions are observed in the shaft response. With the present parameters, no quasi-periodic motion is observed. This figure shows that the chaotic motion is possible in a vast range of eccentricity and rotational speed. From the figure, it is obvious that with increasing the rotational speed or eccentricity, the probability of the occurrence of the chaotic motion increases. One can conclude that in in-extensional rotating shafts, even for a fairly small amount of eccentricity, the chaotic motion is possible. For example, if $e_{t}=0.002$, then chaotic vibration occurs for rotational speed of $\Omega \geq 41.6$. Note that the second critical speed is about $\Omega \approx 40$.

Consequently, for a fairly slender rotating shaft, the chaotic vibration is possible in the rotational speeds above the second critical speed, even for a small amount of eccentricity. For large eccentricity, the chaotic motion appears in the rotational speeds below the second critical speed. There are regions in the diagram, e.g., $e_{t}=0.003$ and $\Omega \approx 40-41.2$, where periodic and chaotic motions occur alternately.

To investigate this diagram more deeply, the timehistory, Poincaré map, bifurcation diagram, Lyapunov exponent, and amplitude spectrum are used. Bifurcation diagrams for the amplitude of the first and secondmode responses in $\nu$-plane, i.e., $\nu_{1}$ and $\nu_{2}$, are plotted against rotational speed $\Omega$ in Figures 3 and 4 . In these 


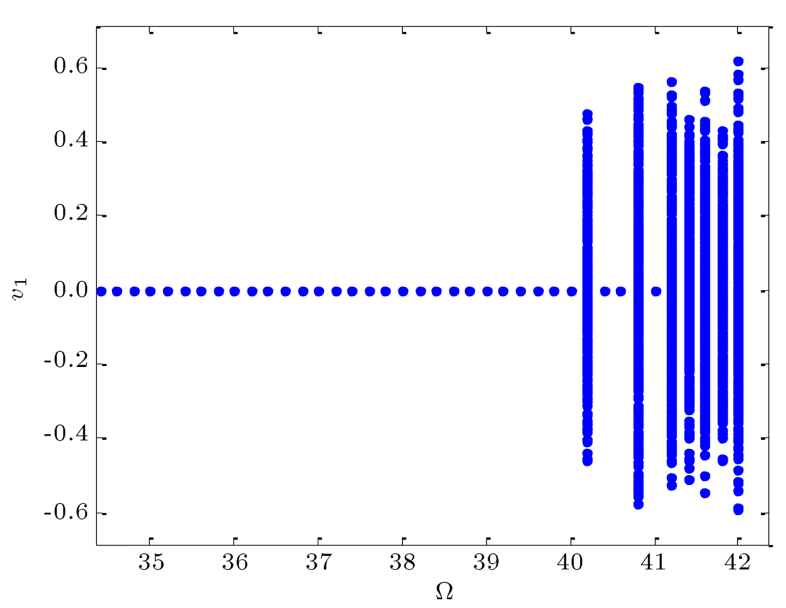

Figure 3. Bifurcation diagram for $v_{1} ; I_{2}=0.000625$, $c=0.03$, and $e_{t}=0.003$.

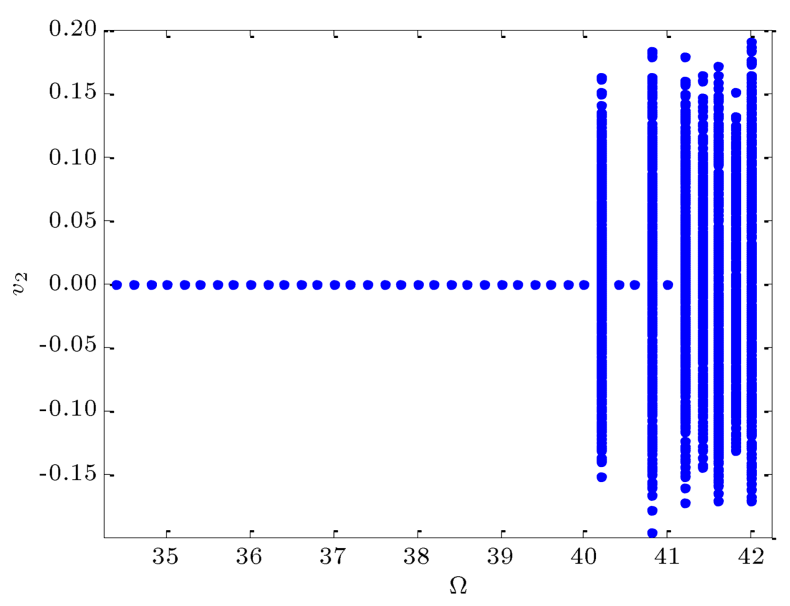

Figure 4. Bifurcation diagram for $v_{2} ; I_{2}=0.000625$, $c=0.03$, and $e_{t}=0.003$.

figures, $e_{t}=0.003$. Note that in these figures, in the range of $\Omega<40.2$, the amplitude of periodic motion is a small value, not zero. The qualitative behavior of both modes is the same. However, amplitude of the secondmode response is smaller. The motion is periodic up to rotational speed of $\Omega \approx 40.2$, where a large-amplitude chaotic motion appears. Above this rotational speed, periodic and chaotic motions occur alternately, and then above $\Omega \approx 41.2$, only chaotic motion is observed. Figures 3-4 show examples of discontinuous bifurcation: the periodic motion suddenly disappears and a chaotic motion occurs.

Time histories and Poincaré map of the first-mode response at $\Omega=40.2$ are shown in Figures 5 and 6 . The aperiodic nature of the chaotic motion is obvious in Figure 5. In periodic response, there exists a single point in Poincaré map. But, Figure 6 shows irregular scattering of points in the plane, and it is a sign of chaos. Figure 7 shows the corresponding amplitude spectrum. When the response is periodic, the spectrum has a single spike in the rotational speed frequency. In

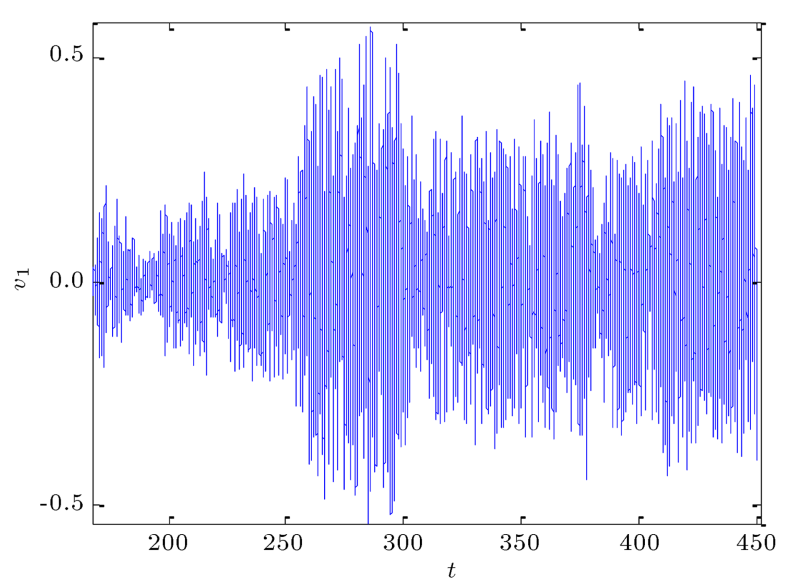

Figure 5. Time history of $v_{1} ; I_{2}=0.000625, c=0.03$, $e_{t}=0.003$, and $\Omega=40.2$.

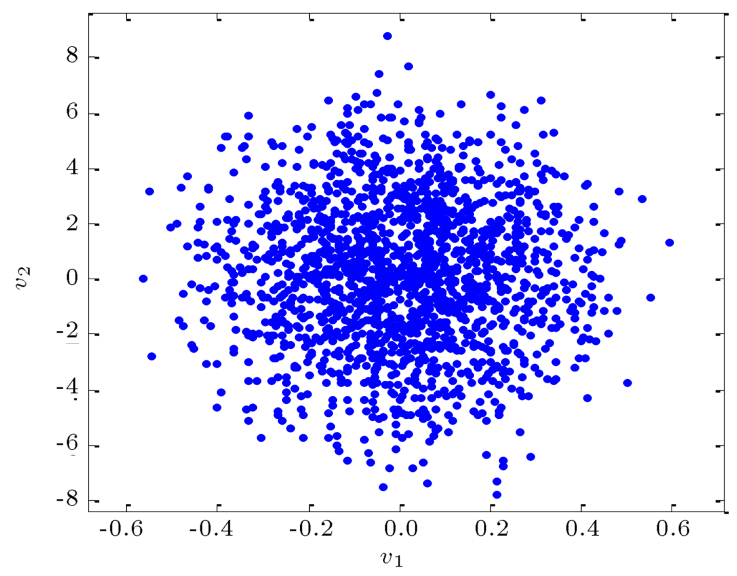

Figure 6. Poincaré map; $I_{2}=0.000625, c=0.03$, $e_{t}=0.003$, and $\Omega=40.2$.

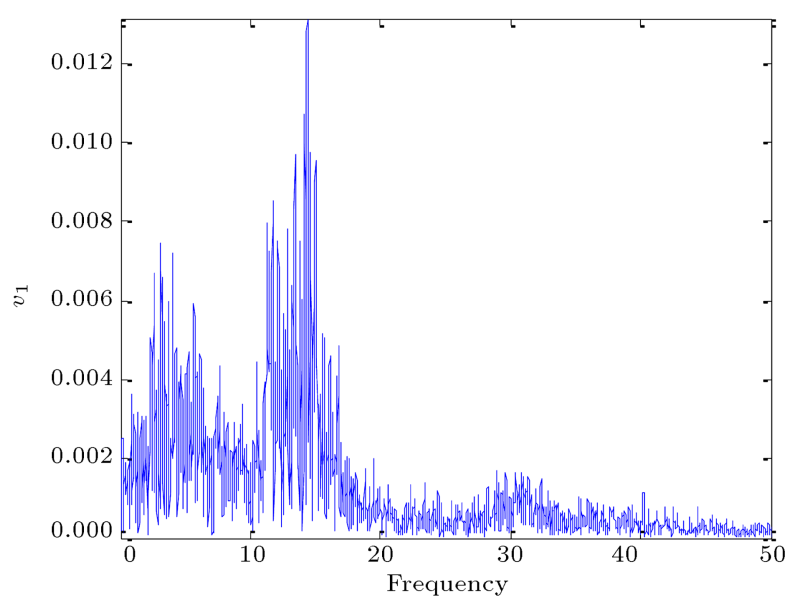

Figure 7. Amplitude spectrum; $I_{2}=0.000625, c=0.03$, $e_{t}=0.003$, and $\Omega=40.2$.

contrast, Figure 7 shows a broadband of frequencies in the response, which is a characteristic of chaotic motion. To quantify the above results, the largest Lyapunov exponent $\lambda$ is computed and plotted against rotational speed in Figure 8 . The largest positive 


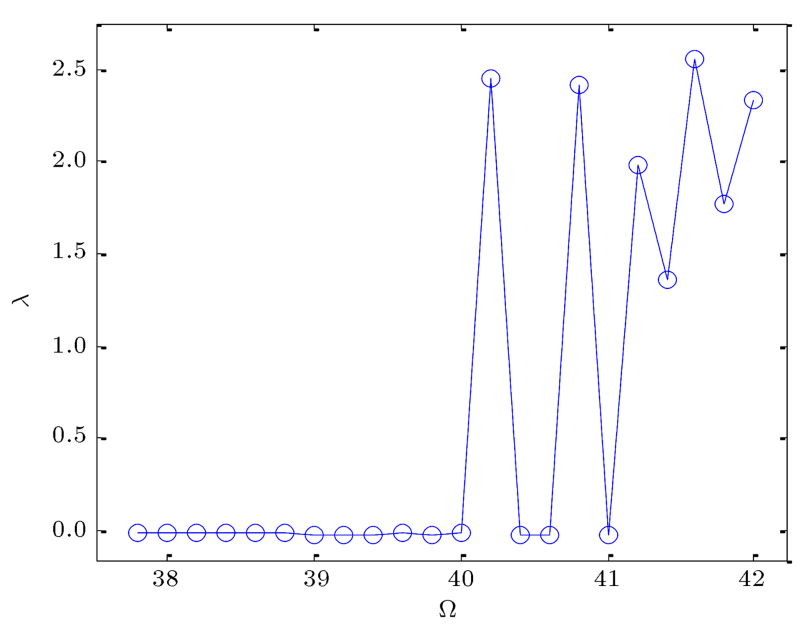

Figure 8. Largest Lyapunov exponent versus rotational speed; $I_{2}=0.000625, c=0.03$, and $e_{t}=0.003$.

Lyapunov exponent $\lambda>0$ denotes a chaotic motion (sensitivity to initial conditions) and a non-positive one $\lambda \leq 0$ shows regular (non-chaotic) motion. Figure 8 shows that a chaotic motion appears first in $\Omega \approx 40.2$, where $\lambda \approx 2.45$.

\subsection{A very slender rotating shaft with negligible gyroscopic effect}

In this sub-section, it is assumed that the shaft is very slender with negligible gyroscopic effect and the dynamical behavior of the shaft is considered. Therefore, it is assumed that $I_{2}=0$ and $c=0.03$. Figure 9 shows different types of motion of the shaft with different eccentricities and rotational speeds. The ranges and steps of eccentricity and rotational speed are same as those in Figure 2. The general trend of the diagram is similar to that in Figure 2. But, in the present shaft with negligible gyroscopic effect, in addition to periodic and chaotic responses, quasi-periodic and transient chaotic motions may occur. Furthermore, the chaotic

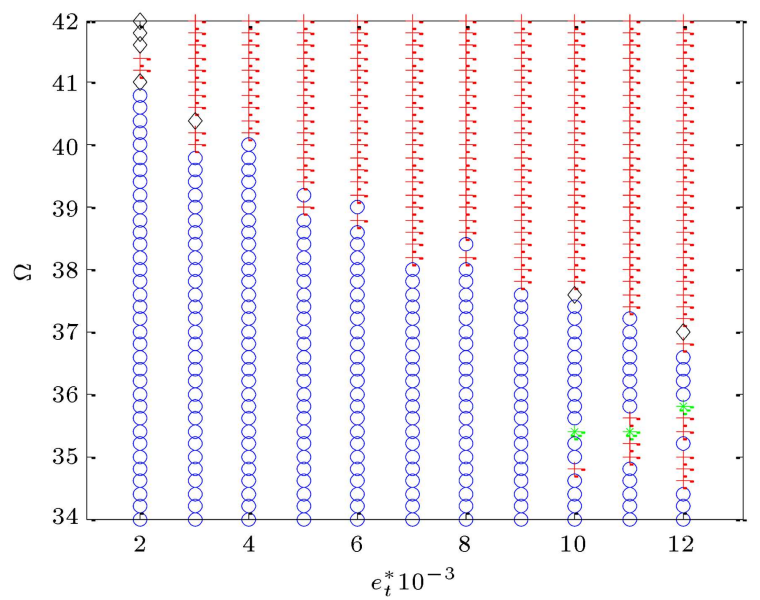

Figure 9. Different types of responses; $I_{2}=0, c=0.03$. $\mathrm{O}$ : periodic, + : chaotic, $\diamond$ : transient chaotic, $*$ : quasi-periodic.

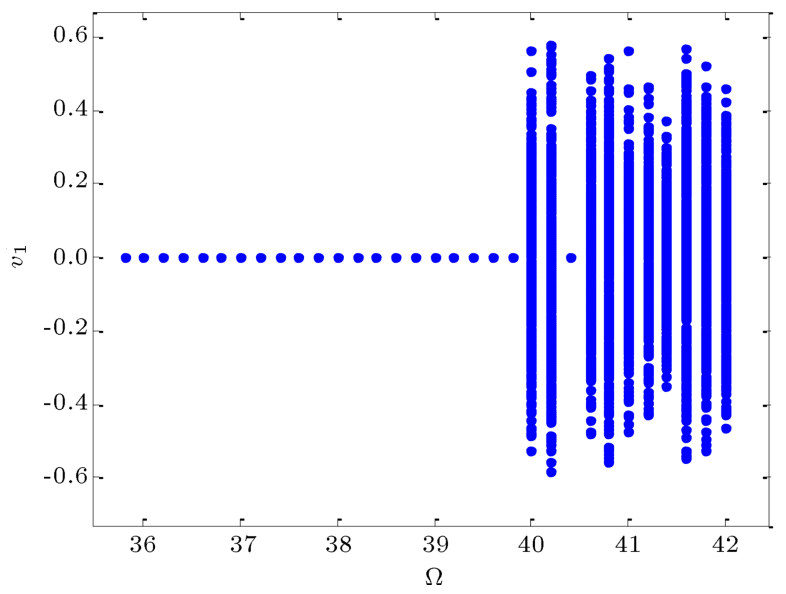

Figure 10. Bifurcation diagram for $\nu_{1} ; I_{2}=0, c=0.03$, and $e_{t}=0.003$.

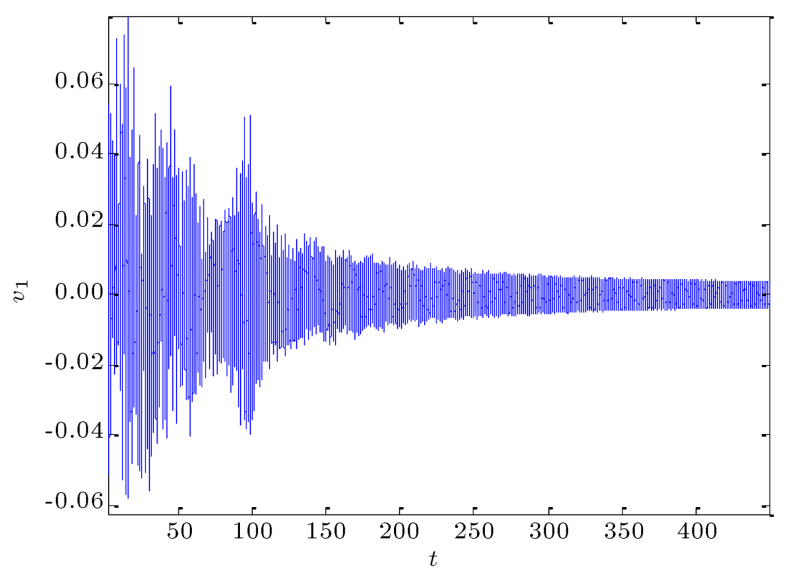

Figure 11. Time history of $\nu_{1} ; I_{2}=0, c=0.03$, $e_{t}=0.003$, and $\Omega=40.4$.

region in this diagram, in comparison with Figure 2, is large. The reason is that when the shaft is very slender, the nonlinearity is bigger and chaos can occur in lower rotational speeds and wider range of parameters.

In the following, this diagram is studied more deeply. Bifurcation diagram for the amplitude of the first-mode response in $\nu$-plane is plotted against rotational speed $\Omega$ in Figure $10\left(e_{t}=0.003\right)$. This figure shows that the motion of the shaft is periodic in rotational speeds below $\Omega \approx 40$. At this rotational speed, a discontinuous bifurcation occurs, and smallamplitude periodic bifurcation disappears and a largeamplitude chaotic motion appears. Up to rotational speed of $\Omega \approx 40.6$, periodic and chaotic motions occur alternately, and then, above this rotational speed, only chaotic motion is observed. At rotational speed of $\Omega \approx 40.4$, "transient chaos" is observed in simulation results (Figure 11). When a transient chaos occurs, in the beginning of the motion, the shaft response is chaotic (the maximum Lyapunov exponent in the beginning portion of the signal is positive), but finally the motion is settled in a periodic attractor and the 


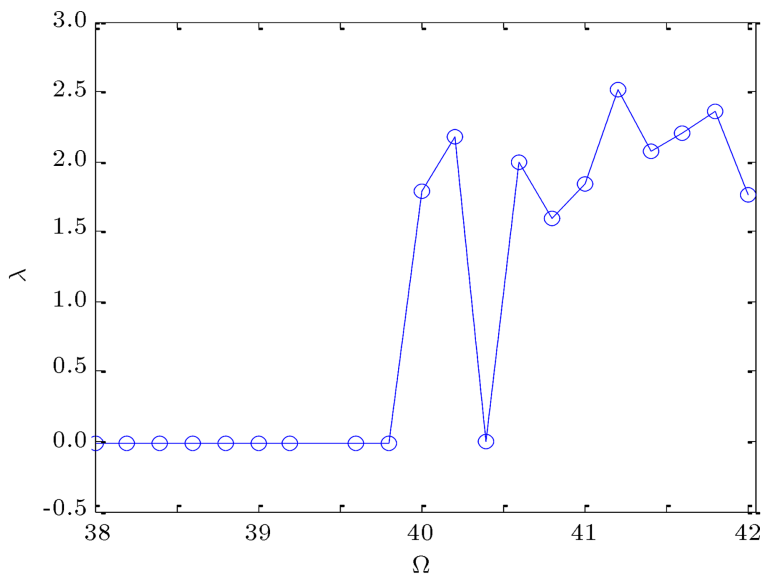

Figure 12. Largest Lyapunov exponent versus rotational speed; $I_{2}=0, c=0.03$, and $e_{t}=0.003$.

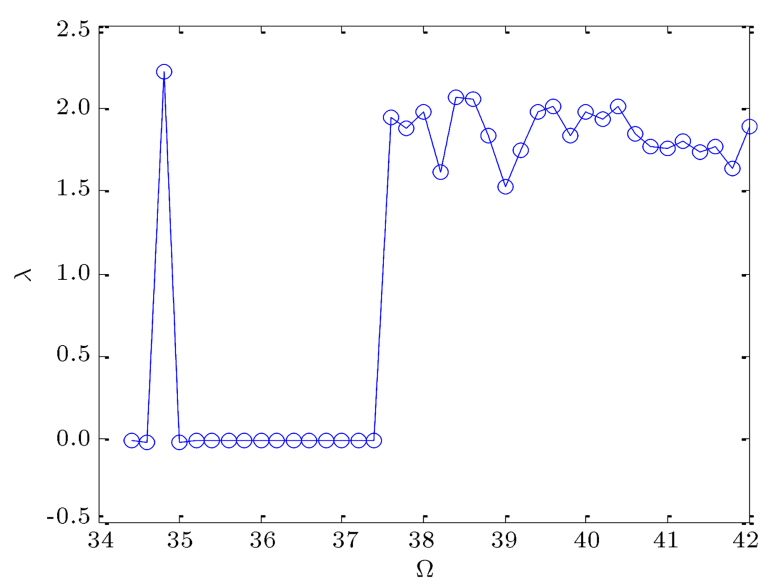

Figure 13. Largest Lyapunov exponent versus rotational speed; $I_{2}=0, c=0.03$, and $e_{t}=0.01$.

asymptotic state of the system is periodic. The largest Lyapunov exponent $\lambda$ is computed and plotted against rotational speed in Figure 12. This diagram shows the chaotic region of the response.

Figure 9 showed that for some values of eccentricity and rotational speed, quasi-periodic motion existed. This case is considered more deeply. The largest Lyapunov exponent $\lambda$ is presented for $e_{t}=0.01$ in Figure 13. For the cases of $\Omega \approx 34.8$ and $\Omega>37.8, \lambda$ is positive and a chaotic motion exists.

From Figure 13, it is observed that the shaft responses for $\Omega=34.6$ and $\Omega=34.8$ are periodic and chaotic, respectively. But, at $\Omega=35.4$ (Figure 14), it seems that the motion is quasi-periodic (the motion is not chaotic because the maximum Lyapunov exponent is not positive). Poincaré map corresponding to Figure 14 is plotted in Figure 15. This figure shows complex closed curves, not a simple closed curve. Thus, the response is not a two-mode quasi-periodic motion, but a multi-mode quasi-periodic one. To investigate more, amplitude spectrum corresponding to this case is plotted in Figure 16. This figure shows that there are

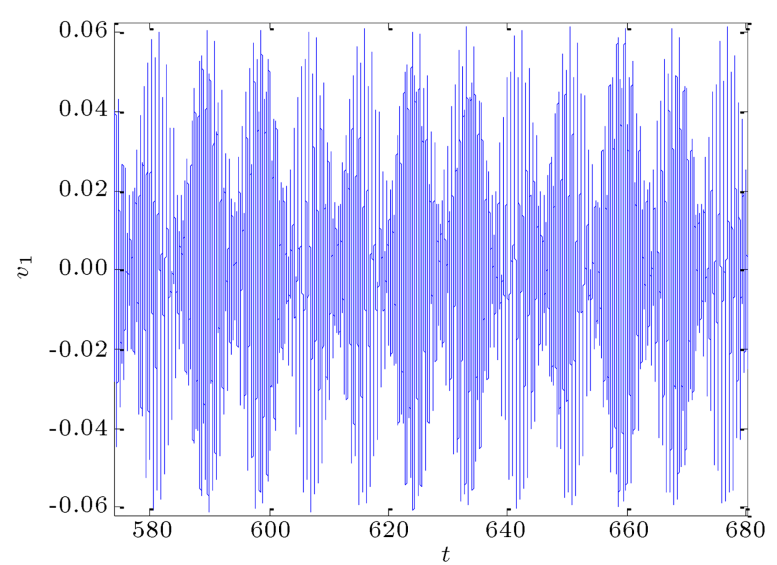

Figure 14. Time history of $\nu_{1} ; I_{2}=0, c=0.03$, $e_{t}=0.01$, and $\Omega=35.4$.

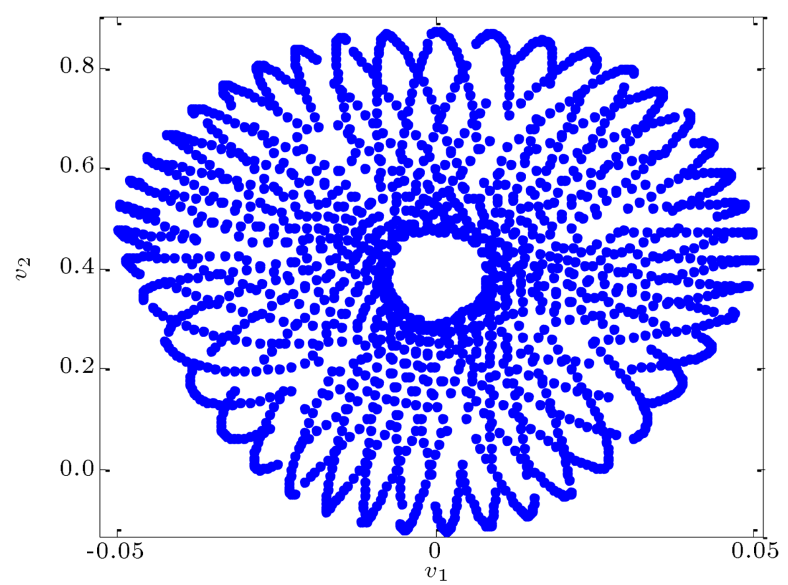

Figure 15. Poincaré map; $I_{2}=0, c=0.03, e_{t}=0.01$, and $\Omega=35.4$.
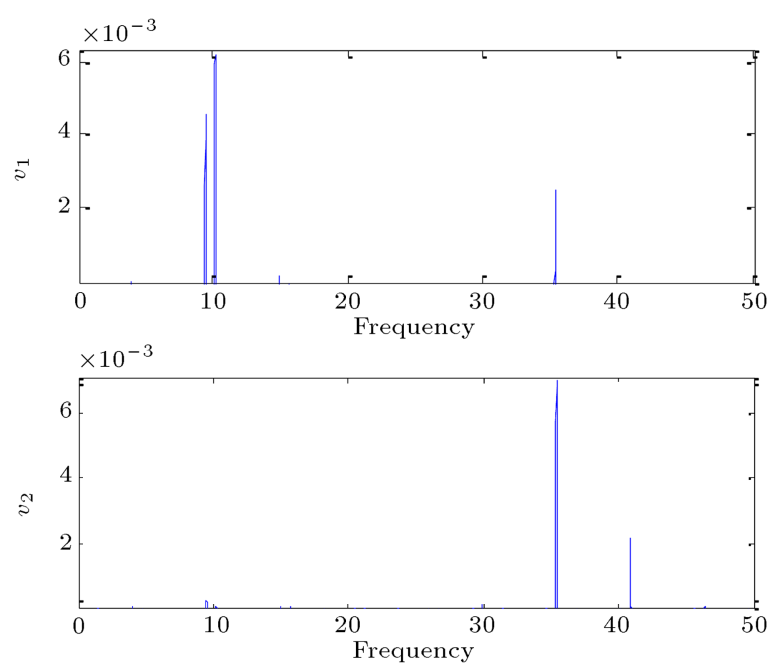

Figure 16. Amplitude spectrum; $I_{2}=0, c=0.03$, $e_{t}=0.01$, and $\Omega=35.4$.

spikes in the spectrum, approximately in frequencies of $9.49,10.21,35.40$, and 40.88 . This confirms that the response is multi-mode quasi-periodic motion. Note that these frequencies have been computed with finite 
precision. With infinite precision, these frequencies would be incommensurate. Otherwise, the motion would be periodic. The frequencies of 9.49 and 10.21 in spectrum are forward and backward first-mode natural frequencies, respectively, and 40.88 is the second forward natural frequency. The frequency of 35.40 is rotational speed frequency. Figure 16 reveals the frequency content of the motion, nicely. Briefly, when the shaft is rotated with the speed of $\Omega=35.4$, it is whiling with three frequencies of 9.49, 10.21, and 40.88 and a quasi-periodic response appears. Note that simulations in the neighborhood of this rotational speed show that this quasi-periodic motion exists only in a narrow region of rotational speed (this result is not presented here).

\subsection{A short lightly damped rotating shaft}

In this subsection, the value of mass moment of inertia is increased and dynamical behavior of the shaft is investigated. Therefore, the shaft is not slender and gyroscopic moment is fairly large. In references $[26,27]$, the authors stated that the chaos occurred in rotors with strong nonlinearity or in rotors with weak asymmetric nonlinearity, but in the presence of internal resonance. Internal resonances occur when the gyroscopic moment is small. Here, the occurrence of chaos in an in-extensional rotor with large gyroscopic moment is observed. In other words, it is shown that the chaotic response is possible for a shaft with weak symmetric nonlinearity without the existence of any internal resonance. Therefore, the present case shows that the chaotic motion can occur in the rotor with wide range of rotary inertia values and it is not necessary that the rotor should have a small gyroscopic moment.

Figure 17 shows different types of motion of the shaft with different eccentricities and rotational

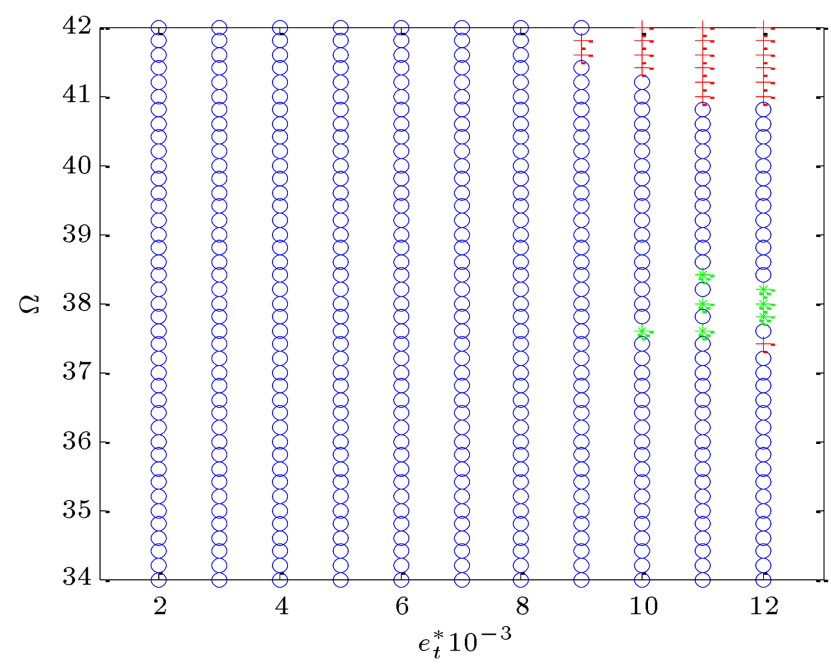

Figure 17. Different types of responses; $I_{2}=0.00626$, $c=0.03$. O: periodic, + : chaotic, $*$ : quasi-periodic. speeds. It is assumed that $I_{2}=0.00625 \mathrm{c}=0.03$. The ranges and steps of eccentricity and rotational speed are same as those in Figure 2. The general trend of the diagram is similar to that in Figures 2 and 9. However, the range of chaotic motion is very small and it occurs in large eccentricity and rotational speed. For eccentricity $e_{t}=0.01$, the largest Lyapunov exponent $\lambda$ is presented in Figure 18. The response is periodic until a discontinuous bifurcation occurs in $\Omega \approx 37.6$, and small-amplitude periodic bifurcation disappears and a fairly-large-amplitude quasi-periodic motion is created. Then, again in $\Omega \approx 37.8$, the response becomes periodic and in a discontinuous bifurcation at $\Omega \approx 41.4$, a large-amplitude chaotic motion appears. The largest Lyapunov exponent shows periodic and chaotic responses in $\Omega=37.4$ and $\Omega=41.4$, respectively. But, the response in $\Omega=37.6$ seems to be quasi-periodic (Figure 19). To

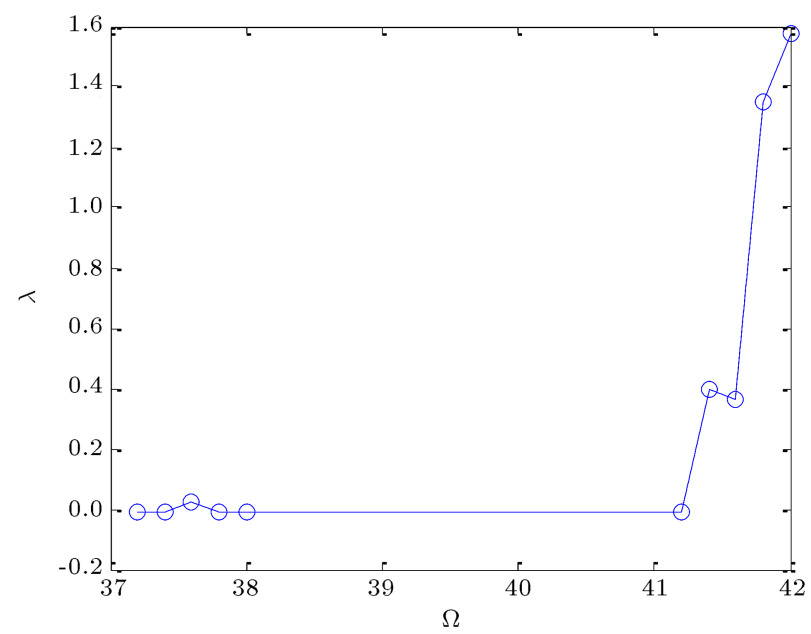

Figure 18. Largest Lyapunov exponent versus rotational speed; $I_{2}=0.00625, c=0.03$, and $e_{t}=0.01$.

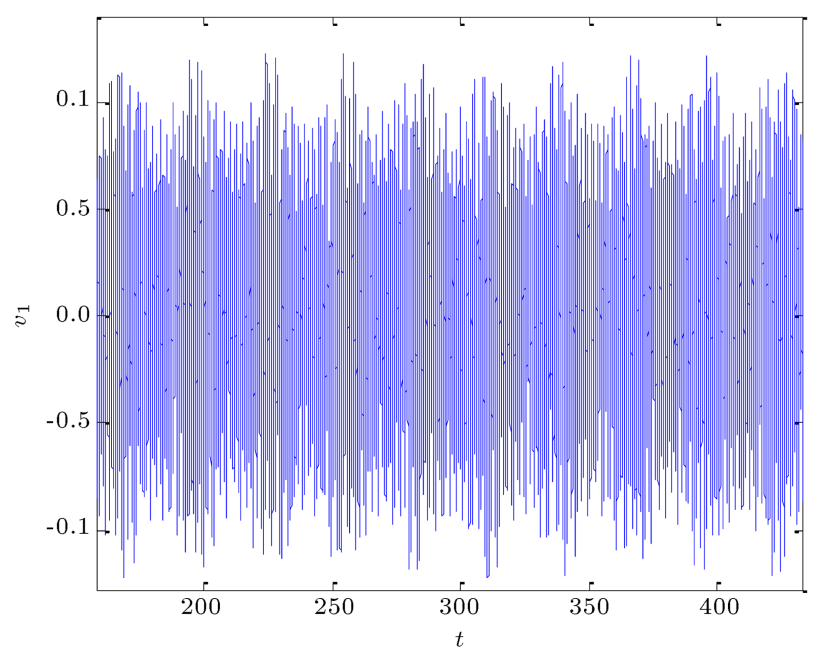

Figure 19. Time history of $\nu_{1} ; I_{2}=0.00625, c=0.03$, $e_{t}=0.01$, and $\Omega=37.6$. 

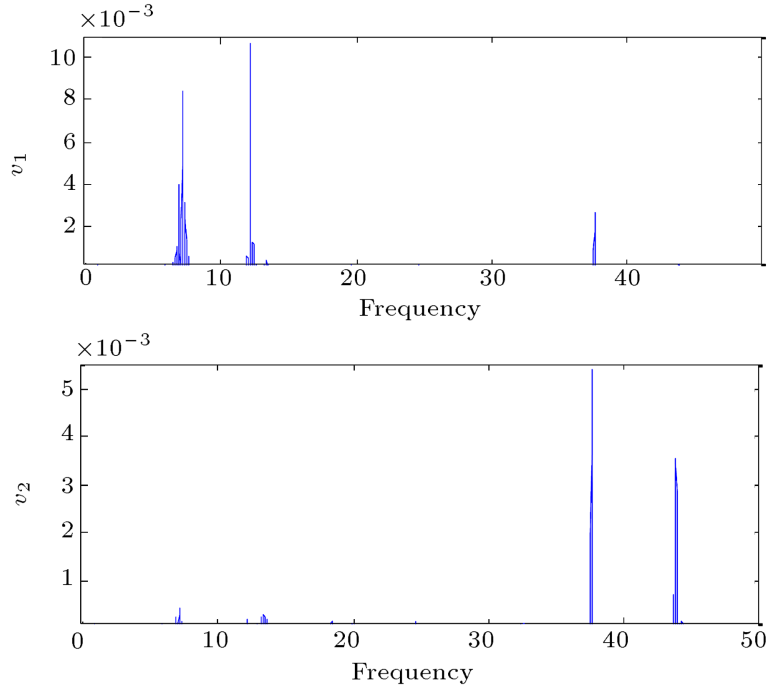

Figure 20. Amplitude spectrum; $I_{2}=0.00625, c=0.03$, $e_{t}=0.01$, and $\Omega=37.6$.

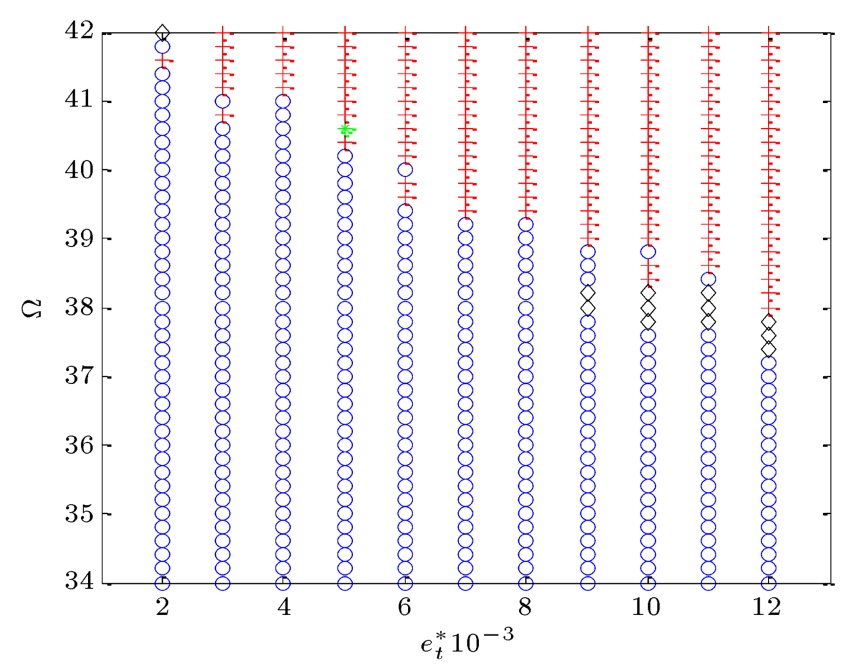

Figure 21. Different types of responses; $I_{2}=0.000625$, $c=0.1$. O: periodic, + : chaotic, $\diamond:$ transient chaotic,

*: quasi-periodic.

investigate more, the amplitude spectrum is plotted for this case in Figure 20. The figure shows that the motion is quasi-periodic. The base frequencies are 7.10, $12.13,37.60$, and 43.85. These frequencies are first forward mode natural frequency, first backward mode natural frequency, second forward natural frequency, and rotational speed frequency, respectively.

\subsection{A slender rotating shaft with high damping}

Now, the effect of damping is considered. Figure 21 shows different types of motion of the shaft with different eccentricities and rotational speeds. The data is the same as that in Figure 2, except that $c=0.1$. In this case, the damping is large. The general trend of the diagram is similar to that of the previous cases. However, the range of chaotic motions is smaller and

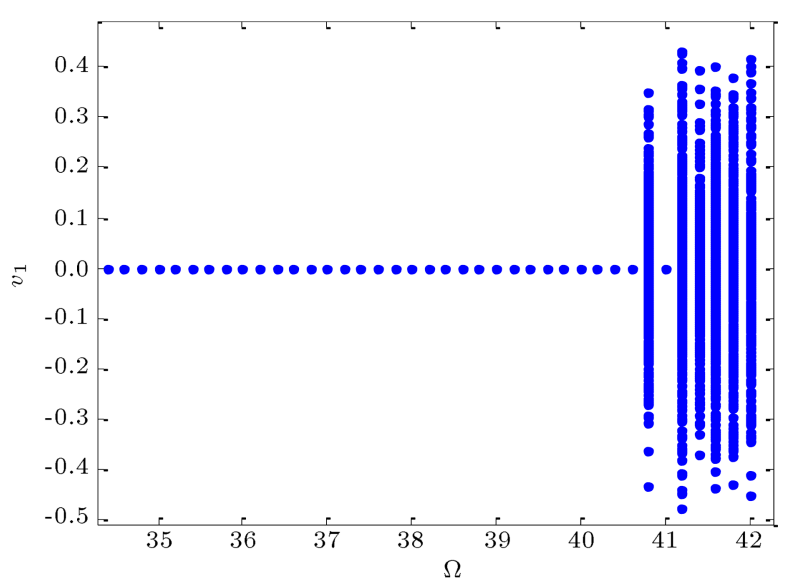

Figure 22. Bifurcation diagram for $\nu_{1} ; I_{2}=0.000625$, $c=0.1$ and $e_{t}=0.003$.

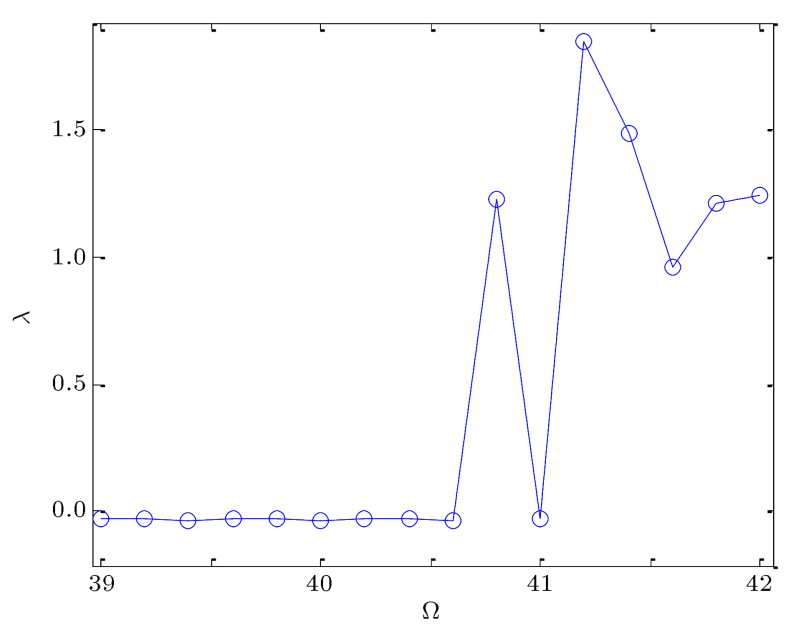

Figure 23. Largest Lyapunov exponent versus rotational speed; $I_{2}=0.000625, c=0.1$ and $e_{t}=0.003$.

transient chaos is observed. For $e_{t}=0.003$, bifurcation diagram of the amplitude of the first-mode response is plotted in Figure 22. The corresponding largest Lyapunov exponent $\lambda$ is presented in Figure 23. These figures show that the response is periodic until a discontinuous bifurcation occurs in $\Omega \approx 40.8$, where smallamplitude periodic motion disappears and a largeamplitude chaotic motion is created. Above this speed, again, periodic solution is observed and with increasing the speed to $\Omega \approx 41.2$, a chaotic motion is created, which is sustained for larger rotational speeds. Other properties of the response are similar to those of the previous cases and are not repeated again.

\section{Conclusion}

In the paper, bifurcation and chaotic dynamics of inextensional rotating shafts were analyzed. Two-mode Galerkin truncation was used to discretize the partial differential equations of motion. The nonlinearity in the system was symmetric in two transverse planes. 
Using numerical integration, it was shown that periodic (synchronous), quasi-periodic, chaotic, and transient chaotic responses were possible for the shaft near the second critical speed. It was proved that the chaotic response existed even for a fairly short rotating shaft. In other words, it was shown that the chaotic response was possible for a shaft with symmetric weak nonlinearity without the existence of any internal resonance. Therefore, one may conclude that the chaotic motion can occur in an in-extensional nonlinear shaft for a wide range of rotary inertia and it is not necessary for the rotor to have a small gyroscopic moment. With some tools from nonlinear dynamics, such as time history, bifurcation diagram, Poincaré map, Lyapunov exponents, and amplitude spectrum, different types of shaft responses were characterized and analyzed. Some results of the paper are as follow:

1. Near the first whirling mode, the shaft response is synchronous;

2. In the neighborhood of the second whirling mode, the rotating shaft shows complex dynamical behavior;

3. When the shaft is slender and the damping is light, chaotic motion is possible in a vast range of eccentricities and rotational speeds;

4. For a fairly slender rotating shaft, even with a small amount of eccentricity, chaotic vibration is possible in the rotational speeds above the second critical speed. For large eccentricities, the chaotic motion appears in the rotational speeds below the second critical speed;

5. In the rotating shaft with negligible gyroscopic effect, in addition to periodic and chaotic motions, quasi-periodic motion and transient chaos are observed. Furthermore, the chaotic region occurs in a broader range of parameters;

6. Chaos can occur in the in-extensional rotating shaft with fairly large amounts of damping and gyroscopic moment.

\section{Acknowledgement}

The study was performed in Faculty of Engineering at Kharazmi University under a grant presented by the Vice Chancellor of Research, which is acknowledged.

\section{References}

1. Ishida, Y. "Review of research on nonlinear rotordynamics in Japan", Journal of System Design and Dynamics, 7, pp. 151-169 (2013).

2. Ehrich, F. "Some observations of chaotic vibration phenomena in high-speed rotor dynamics", ASME Journal of Vibration and Acoustics, 113, pp. 50-57 (1991).
3. Muszynska, A. and Goldman, P. "Chaotic responses of unbalanced rotor/bearing/stator systems with looseness or rub", Chaos, Solitons, and Fractals, 5, pp. 1683-1704 (1995).

4. Chu, F.L. and Zhang, Z. "Periodic, quasi-periodic and chaotic vibrations of a rub-impact rotor system supported on oil film bearings", International Journal of Engineering Science, 35, pp. 963-973 (1997).

5. Chu, F.L. and Zhang, Z. "Bifurcation and chaos in a rub-impact Jeffcott rotor system", Journal of Sound and Vibration, 210, pp. 1-18 (1998).

6. Dai, X., Zhang, X., and Jin, X. "The partial and full rubbing of a flywheel rotor-bearing-stop system", International Journal of Mechanical Science, 43, pp. 505-519 (2001).

7. Sun, Z., Xu, J., and Zhou, T. "Analysis on complicated characteristics of a high-speed rotor system with rubimpact", Mechanism and Machine Theory, 37, pp. 659-672 (2002).

8. Luo, Y., Zhang, S., and Wen, B. "Dynamic characteristics of nonlinear elastics rotor system with rub-impact faults", Turbine Technology, 46, pp. 415-417 (2004).

9. Zhang, W.M. and Meng, G. "Stability, bifurcation and chaos of a high-speed rub-impact rotor system", Sensors and Actuators A, 127, pp. 163-78 (2006).

10. Yuan, Z., Chu, F., and Hao, R. "Simulation of rotor's axial rub-impact in full degrees of freedom", Mechanism and Machine Theory, 42, pp. 763-775 (2007).

11. Shen, X., Jia, J., and Zhao, M. "Nonlinear analysis of a rub-impact rotor-bearing system with initial permanent rotor bow", Archive of Applied Mechanics, 78, pp. 225-240 (2008).

12. Khanlo, H.M., Ghayour, M., and Ziaei-Rad, S. "Chaotic vibration analysis of rotating, flexible, continuous shaft-disk system with a rub-impact between the disk and the stator", Commun Nonlinear Sci Numer Simulat, 16, pp. 566-582 (2011).

13. Patel, T.H., Zuo, M.J., and Zhao, X. "Nonlinear lateral-torsional coupled motion of a rotor contacting a viscoelastically suspended stator", Nonlinear Dynamics, 69, pp. 325-339 (2012).

14. Wang, J., Zhou, J., Dong, D., Yan, B., and Huang, C. "Nonlinear dynamic analysis of a rub-impact rotor supported by oil film bearings", Archive of Applied Mechanics, 83, pp. 413-430 (2013).

15. Varney, P. and Green, I. "Nonlinear phenomena, bifurcations, and routes to chaos in an asymmetrically supported rotor-stator contact system", Journal of Sound and Vibration, 336, pp. 207-226 (2015). 
16. Xiang, L., Hu, A., Hou, L., et al. "Nonlinear coupled dynamics of an asymmetric double-disc rotor-bearing system under rub-impact and oil-film forces", Applied Mathematical Modelling, 40, pp. 4505-4523 (2016).

17. Yang, Y., Xu, Y., Yang, Y., et al. "Dynamics characteristics of a rotor-casing system subjected to axial load and radial rub", International Journal of Non-Linear Mechanics (2017) DOI: 10.1016/j.ijnonlinmec.2017.10.023

18. Jian, C-W.C. and Chen, C-K. "Bifurcation and chaos analysis of a flexible rotor supported by turbulent long journal bearings", Chaos, Solitons and Fractals, 34, pp. 1160-1179 (2007).

19. Jian, C-W.C. and Chen, C-K. "Chaotic response and bifurcation analysis of a flexible rotor supported by porous and non-porous bearings with nonlinear suspension", Nonlinear Analysis: Real World Applications, 10, pp. 1114-1138 (2009).

20. Wang, C.-C. "Bifurcation and nonlinear analysis of a flexible rotor supported by a relative short spherical gas bearing system", Communications in Nonlinear Science and Numerical Simulation, 15, pp. 2659-5571 (2010).

21. Cháveza, J.P. and Wiercigrocha, M. "Bifurcation analysis of periodic orbits of a non-smooth Jeffcott rotor Model", Communications in Nonlinear Science and Numerical Simulation, 18, pp. 2571-2580 (2013).

22. Dakel, M., Baguet, S., and Dufour, R. "Nonlinear dynamics of a support-excited exible rotor with hydrodynamic journal bearings", Journal of Sound and Vibration, 333, pp. 2774-2799 (2014).

23. Yan, S., Dowell, E.H., and Lin, B., "Effects of nonlinear damping suspension on nonperiodic motions of a flexible rotor in journal bearings", Nonlinear Dynamics, 78, pp. 1435-1450 (2014).

24. Chen, J.H. and Wang, C.C. "Chaotic and dynamic analysis of a flexible rotor supported by ultra short aero-lubricated bearing system", Journal of Applied Research and Technology, 13, pp. 328-341 (2015).

25. Przybylowicz, P.M., Starczewski, Z., and KorczakKomorowski, P. "Sensitivity of regions of irregular and chaotic vibrations of an asymmetric rotor supported on journal bearings to structural parameters", Acta Mechanica, 227, pp. 3101-3112 (2016).

26. Yamamoto, T. and Ishida, Y., Linear and Nonlinear Rotordynamics: A Modern Treatment with Applications. Wiley-Interscience (2001).

27. Inoue, T. and Ishida, Y. "Chaotic vibration and internal resonance phenomena in rotor systems", $J$. Vib. Acoust., 128, pp. 156-169 (2006).
28. Nagasaka, I., Liu, J., and Ishida Y. "Forced vibrations of a very slender continuous rotor with geometrical nonlinearity (harmonic and subharmonic resonances)", J. Vib. Acoust., 132, 021004, 9 pages (2010).

29. Hosseini, S.A.A. and Khadem, S.E. "Free vibrations analysis of a rotating shaft with nonlinearities in curvature and inertia", Mech. Mach. Theory, 44, pp. 272-288 (2009).

30. Hosseini, S.A.A. and Khadem, S.E. "Combination resonances in a rotating shaft", Mech. Mach. theory, 44, pp. 1535-1547 (2009).

31. Khadem, S.E., Shahgholi, M., and Hosseini, S.A.A. "Primary resonances of a nonlinear in-extensional rotating shaft", Mech. Mach. Theory, 45, pp. 1067-1081 (2010).

32. Khadem, S.E., Shahgholi, M., and Hosseini, S.A.A. "Two-mode combination resonances of an in-extensional rotating shaft with large amplitude", Nonlinear Dynam., 65, pp. 217-233 (2011).

33. Nayfeh, A.H. and Pai, P.F., Linear and Nonlinear Structural Mechanics, Wiley-Interscience, New York (2004).

34. Argyris, J., Faust, G., and Haase, M., An Exploration of Chaos, North-Holland (1994).

35. Sadoudi, S., Tanougast, C., Azzaz, M.S., et al. "Design and FPGA implementation of a wireless hyperchaotic communication system for secure real-time image transmission", EURASIP Journal on Image and Video Processing, 43, pp. 1-18 (2013).

\section{Appendix}

To solve the differential equations of motion, the fourth-order Runge-Kutta method is used. Eq. (5) can be written in the state space form as:

$$
\begin{aligned}
\dot{X}_{i}= & F_{i}\left(X_{1}, X_{2}, X_{3}, X_{4}, X_{5}, X_{6}, X_{7}, X_{8}, t\right), \\
& i=1-8,
\end{aligned}
$$

where:

$$
\begin{array}{ll}
X_{1}=\nu_{1}, & X_{2}=\dot{\nu}_{1}, \\
X_{3}=\nu_{2}, & X_{4}=\dot{\nu}_{2}, \\
X_{5}=w_{1}, & X_{6}=\dot{w}_{1}, \\
X_{7}=w_{2}, & X_{8}=\dot{w}_{2},
\end{array}
$$

and $F_{i}(i=1-8)$ are nonlinear functions.

Initial conditions (i.e., variable $X_{i}$ in the 0 th iteration) are denoted by $X_{i, 0}(i=1-8)$. The flowchart of the fourth-order Runge-Kutta method is shown in Figure A.1 [35]. In this figure, $h$ is iteration step and $k_{i, j}$ is the $i$ th intermediate slope $(i=0-3)$ for the $j$ th equation $(j=1-8)$. 


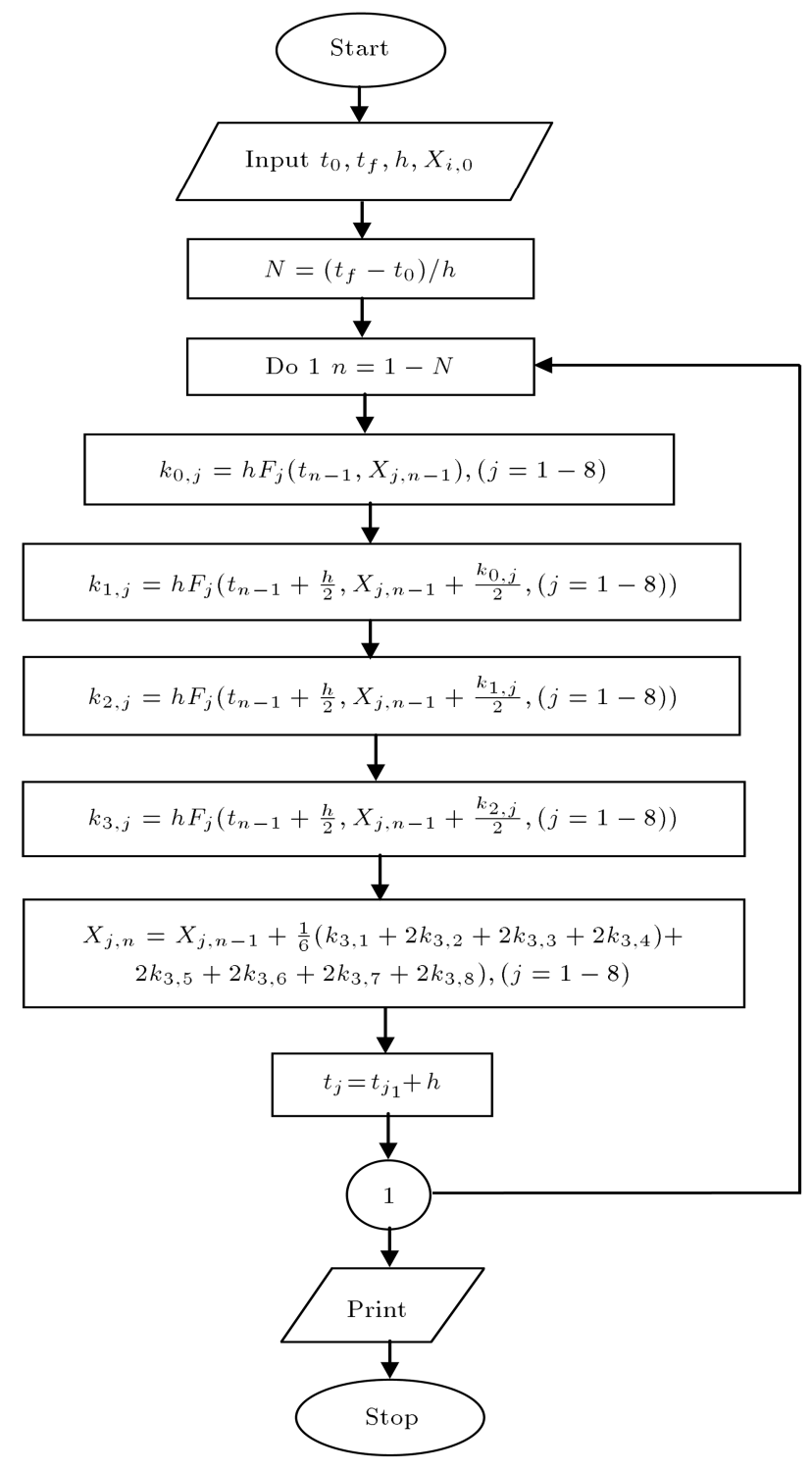

Figure A.1. The flowchart of the fourth-order Runge-Kutta method.

\section{Biography}

Seyyed Ali Asghar Hosseini was born in Qom, Iran, in 1978. He received his BS degree from Iran University of Science and Technology, and MS and PhD degrees from Tarbiat Modares University, Tehran, Iran. He is currently Associate Professor in the Mechanical Engineering Department of Kharazmi University, Tehran, Iran. His research interests include nonlinear vibrations, rotor dynamics, and random vibrations. 